\title{
Stability of the PBLG Helix at High Temperature and the Wormlike Character of the Helix
}

\author{
Kinko Tsuji, Hidefusa OHe, and Hiroshi Watanabe \\ Department of Chemistry, College of General \\ Education, University of Tokyo, \\ Meguro-ku, Tokyo, Japan.
}

(Received September 30, 1972)

\begin{abstract}
Viscosity and electric birefringence of poly( $\gamma$-benzyl-L-glutamate) (PBLG) in $m$-cresol have been measured up to $200^{\circ} \mathrm{C}$ (viscosity) and up to $170^{\circ} \mathrm{C}$ (electric birefringence), respectively. Dipole moments, optical anisotropy factors, mean relaxation times of birefringence, and intrinsic viscosities of PBLG at various temperatures have been determined. No abrupt changes which suggest breakage of the helix structure have been observed on hydrodynamical and electrooptical properties. However, the PBLG helix becomes considerably more flexible with increasing temperature. Persistence lengths of PBLG helix at various temperatures have been estimated from dipole moments, relaxation times, and intrinsic viscosity data.
\end{abstract}

KEY WORDS Electric Birefringence / Poly $\gamma$-benzyl-L-glutamate) / Wormlike Chain / Persistence Length /

It is well established that poly $(\gamma$-benzyl-Lglutamate) (PBLG) undergoes temperature-induced helix - coil transition in mixed solvents. ${ }^{1}$ In this case, dichloroacetic acid, trifluoroacetic acid, and hydrazine act as helix breaking reagents and the helix conformation is more stable at higher temperatures. It is also well known that, in contrast to PBLG, poly( $\beta$-benzyl-L-aspartate) (PBLA) undergoes a normal helix-coil transition in $m$-cresol in the temperature range from 30 to $60^{\circ} \mathrm{C} .^{2}$

It may be worthwhile, therefore, to investigate the stability of PBLG helix in $m$-cresol at high temperatures. If one could observe the transition, one could estimate the effect of side chains of PBLG on the stability of the helix. On the other hand, if one could confirm the helical conformation of PBLG at high temperatures, it would give further information with respect to the stability of the PBLG helix.

Franzen, et al., ${ }^{3}$ have investigated the conformation of PBLG at high temperatures. They measured the ORD of PBLG in chloronaphthalene up to $168^{\circ} \mathrm{C}$ and in $N$-methyl caprolactame up to $157^{\circ} \mathrm{C}$, and observed a significant decrease in the Moffitt-Yang $b_{0}$ values at the upper temperature limits of the study. They interpreted the significant decrease in $b_{0}$ at the upper temperature limits as a beginning of helix to coil transition. However, polymer degradation at high temperatures prevented them from verifying the transition.

In order to investigate the stability of the helical conformation of PBLG in $m$-cresol at high temperatures, the authors measured the viscosity up to $200^{\circ} \mathrm{C}$, and the electric birefringence up to $170^{\circ} \mathrm{C}$ by the use of rectangular pulse technique. ${ }^{4,5}$ From the saturation curve of electric birefringence one can determine the dipole moment and optical anisotropy factor of the solute molecule separately within a limit of appropriate assumptions. At the same time, the relaxation time for the decay of birefringence, after instantaneous removal of an applied electric field, was determined. It is sensitive to the change of flexibility of helix to coil conformational change. Thus, the transient electric birefringence method is a powerful tool for investigating the helical molecule in solution at high temperature.

The results show that, in $m$-cresol, PBLG retains its helical structure up to $200^{\circ} \mathrm{C}$, but the helix becomes considerably more flexible, that is, wormlike behaviour of the helix becomes a 
distinguishing feature of PBLG.

\section{EXPERIMENTAL PROCEDURE}

\section{Materials}

A PBLG sample was prepared by the BloutKarlson method. ${ }^{6} \quad \gamma$-Benzyl- $N$-carboxy-L-glutamate anhydride was polymerized in dioxane solution with triethylamine initiator. Its molecular weight was found to be $2.1 \times 10^{5}$ from the intrinsic viscosity in dimethylformamide. ${ }^{7}$

Dimethylformamide (DMF) was dried over $\mathrm{KOH}$ pellets and fractionally distilled at $50 \mathrm{mmHg}$. Ethylene dichloride (EDC) was dried over $\mathrm{KOH}$ pellets and fractionally distilled.

$m$-Cresol was dried with phosphorus pentoxide, and fractionally distilled at $7 \mathrm{mmHg}, 77^{\circ} \mathrm{C}$.

\section{Viscosity Measurements}

Viscosity measurements were made with an Ubbelohde viscometer in which the solvent has a flow time of over $100 \mathrm{sec}$. The temperature was controlled within $\pm 0.05^{\circ} \mathrm{C}$.

\section{Electric Birefringence Measurements}

The electric birefringence apparatus was similar to that described previously. ${ }^{8,9}$ In order to eliminate effects due to the residual birefringence of the cell, a Babinet-Soleil compensator (BSC) was inserted as a birefringence plate between the Kerr cell and the analyzer. Its optical axis was parallel or perpendicular to the direction of the electric field in solution. The polarizer was set at $45^{\circ}$ with reference to the electric field, and the analyzer was set crossed to the polarizer. Adjusting a microscrew attached to BSC, one can select a suitable magnitude of optical retardation due to BSC. Let $\delta_{0}$ be the optical retardation due to $\mathrm{BSC}$, the intensity of light at the photomultiplier is given by

$$
I=I_{0} \sin ^{2}\left(\delta_{0} / 2\right)+I_{\mathrm{s}}
$$

where $I_{0}$ is the intensity of light which would have reached the photomultiplier if the polarizer and the analyzer were parallel and $\delta_{0}$ equals zero, not including the stray light, and $I_{\mathrm{s}}$ is the intensity of stray light.

When an electric field is applied, the solution becomes birefringent, and produces optical retardation $\delta$. Then, the intensity of light at the photomultiplier becomes

$$
I^{\prime}=I_{0} \sin ^{2}\left(\frac{\delta_{0}+\delta}{2}\right)+I_{\mathrm{s}}
$$

Therefore, the change in the intensity of light at the photomultiplier produced by the electric field, $\Delta I$, is given by

$$
\Delta I=I^{\prime}-I=I_{0} \sin \frac{\delta}{2} \sin \left(\delta_{0}+\frac{\delta}{2}\right)
$$

As can be seen from eq 3, insertion of BSC significantly improves the sensitivity of the optical system. Moreover, one can determine the sign of $\delta$. An iterative procedure was programmed to determine the value of $\delta$ from the values of $\Delta I / I_{0}$ and $\delta_{0}$.

The Kerr cell was held in a metal jacket through which paraffin oil was circulated from a constant temperature bath. A Beckmann-type spectrometer cell was used as the Kerr cell. The electrodes of bright platinum supported by Teflon plates were spaced $0.176 \mathrm{~cm}$ apart.

A xenon arc lamp $(150 \mathrm{~W})$ powered by a stabilized D.C. supply was used as a light source. Two interference filters were used to monochromatize the light. Its mean wavelength was $5470 \AA$, and half width was $30 \AA$.

All the measurements of electric birefringence, except those for concentration dependence, were performed at fixed concentration, $c=1.0 \mathrm{~g} / l$.

\section{RESULTS}

\section{Characterization of PBLG Sample by Electric} Birefringence and Viscosity Measurements

From the electric field dependence of the birefringence of PBLG in DMF, the average dipole moment and the average optical anisotropy factor of PBLG were determined. Under the electric field used in this experiment, DMF itself became noticeably birefringent. Its Kerr constant was found to be $7.56 \times 10^{-6} \mathrm{~cm}^{-1} \mathrm{esu}^{-2}$ at $25^{\circ} \mathrm{C}$ and $\lambda=5470 \AA$.

In this procedure, it is assumed that a PBLG molecule in DMF is a rigid rod with a large permanent dipole moment $\mu$ along the molecular axis. Then the birefringence $\Delta n$ of the solution, subtracted by the birefringence due to DMF, can be expressed by a function ${ }^{10}$

$$
\Delta n=\frac{2 \pi c\left(g_{1}-g_{2}\right)}{n \rho}\left[1-3\left(\operatorname{coth} \beta-\frac{1}{\beta}\right) / \beta\right]
$$


Stability of PBLG at High Temperature

Table I. Some characteristic values of PBLG in DMF and EDC

\begin{tabular}{cccccccc}
\hline & {$[\eta]^{25^{\circ} \mathrm{C}}, 1 / \mathrm{d} l$} & $(B / c)_{\substack{E \rightarrow 0 \\
C \rightarrow 0}}$, cgs esu & $\left(g_{1}-g_{2}\right) \times 10^{3}, \mathrm{~cm}^{3}$ & $\mu \times 10^{-3}, D$ & $(l)_{C \rightarrow 0}, \AA$ & $\mu / l, D / \AA$ & $\mu /$ resid, $D$ \\
\hline DMF & 3.48 & $0.150 \pm 0.005$ & 4.30 & 2.75 & $1200 \pm 50$ & $2.2 \pm 0.05$ & 3.3 \\
EDC & 4.2 & $0.190 \pm 0.005$ & 4.64 & 3.4 & 1440 & 2.36 & 3.5 \\
\hline
\end{tabular}

with

$$
\beta=\mu E / k T
$$

where $\pi, k$, and $T$ have their usual meanings, $E$ is the field strength, $n$ is the refractive index of the solution, $\rho$ is the density of the solute, $c$ is the concentration of PBLG expressed in $\mathrm{g} / \mathrm{cm}^{3}, g_{1}-g_{2}$ is the optical anisotropy factor. Equation 4 implies that the contribution due to the induced dipole moment to the electric birefringence is neglected, which is justified in this case.

Since full saturation of $\Delta n$ could not be attained, trial and error methods have been used to determine the best values of $\mu$ and $g_{1}-g_{2}$ from the experimental curves of $\Delta n v s$. $E^{2} / k T$.

The relaxation times $\bar{\tau}$ at several concentrations have been determined by the area method, ${ }^{11,12}$ and extrapolated to obtain the limiting value $(\tau)_{\substack{C \rightarrow 0 \\ E \rightarrow 0}}$ Broersma's equation for the rotational relaxation time of rigid cylindrical rod yields ${ }^{13}$

$$
\begin{aligned}
l=2 & {\left[\frac{9 k T(1 / \bar{\tau})^{-1}}{4 \pi \eta_{0}}\{\ln 2 p-1.57\right.} \\
& \left.\left.+7\left(\frac{1}{\ln 2 p}-0.28\right)^{2}\right\}\right]^{1 / 3}
\end{aligned}
$$

where $p$ is the ratio of the length $l$ to the diameter $d$ of the cylinder and $\eta_{0}$ is the viscosity of medium. Assuming the diameter to be $15 \AA$, we calculated the average molecular length by this equation.

Table I summarizes the results along with the dipole moments per unit length and per residue. The limiting values of the specific Kerr constant $(B / c)_{C \rightarrow 0}=\left(\Delta n / \lambda E^{2}\right)_{\substack{C \rightarrow 0 \\ E \rightarrow 0}}$ are also included in Table I. In the third row, corresponding values obtained in EDC solutions are presented. As can be seen, the intrinsic viscosity, the optical anisotropy factor, the dipole moment and the average length of PBLG are greater in EDC than in DMF. These results strongly sug- gest that PBLG partially associates head-to-tail in EDC.

By using Simha's equation for the intrinsic viscosity of rigid rod $^{14}$

$$
\eta_{\mathrm{sp}}=0.207(l / d)^{1.732} c \phi
$$

one can calculate the length of the molecule. In this equation, $\phi$ is the specific volume of solute. Letting $d=15 \AA$ and $\phi^{-1}=1.27,{ }^{15}$ one obtains $l=1260 \AA$. This result supports the treatment of the data of electric birefringence based on the assumption that the PBLG molecule can be regarded as a rigid dipole, although it may be regarded as a weakly bending rod.

\section{Temperature Dependence of Viscosity}

Temperature dependence of the specific viscosity of PBLG in $m$-cresol is shown in Figure 1. The specific viscosity decreases almost linearly with increasing temperature. When the solution was cooled after heating up to $200^{\circ} \mathrm{C}$, the viscosity increased back to its original value at each temperature.

In Figure 2, the temperature dependence of intrinsic viscosity of PBLG in $m$-cresol is shown. It also decreased remarkably with increasing

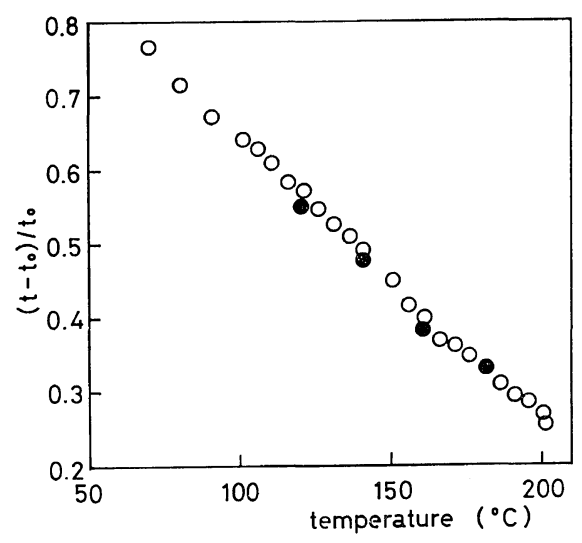

Figure 1. Temperature dependence of specific viscosity of PBLG in $m$-cresol solution: $\bigcirc$, temperature increased; - temperature decreased. 




Figure 2. Temperature dependence of intrinsic viscosity of PBLG in $m$-cresol solution.

temperature, but did not show any abrupt change. Electric Birefringence

Figure 3 shows field strength dependences of the electric birefringence of PBLG (expressed as optical retardation $\delta$ ) in $m$-cresol at various temperatures, where the contributions from $m$ cresol have been subtracted. Full lines are theoretical curves calculated by eq 4 . Values of optical anisotropy factor and permanent dipole moment at each temperature were determined so as to produce the best fit between experimental and theoretical values. They are summarized in Table II.

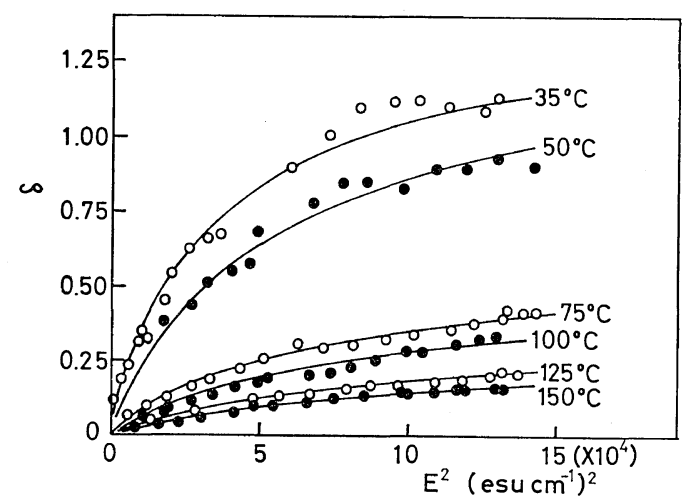

Figure 3. Field strength dependences of electric birefringence (expressed in optical retardation) of PBLG in $m$-cresol solution at various temperatures. Full lines are the theoretical curves for rigid dipole.
Table II. Specific Kerr constant, dipole moment, optical anisotropy factor, and relaxation time of PBLG in $m$-cresol at various temperatures

\begin{tabular}{ccccc}
\hline $\begin{array}{c}\text { Temp, } \\
{ }^{\circ} \mathrm{C}\end{array}$ & $\begin{array}{c}B / c, \\
\text { cgs esu }\end{array}$ & $\mu, D$ & $\begin{array}{c}\left(g_{1}-g_{2}\right) \times 10^{3}, \bar{\tau} / \eta_{0} \times 10^{3}, \\
\mathrm{~cm}^{3}\end{array}$ & \begin{tabular}{c} 
cgs \\
\hline 35
\end{tabular} $0^{3}$ \\
50 & 0.105 & 2260 & 4.70 & 5.78 \\
75 & 0.0186 & 2140 & 4.64 & 5.69 \\
100 & 0.0102 & 1930 & 2.17 & 6.60 \\
125 & 0.00679 & 1740 & 1.79 & 2.98 \\
150 & 0.00340 & 1610 & 1.25 & 3.12 \\
170 & - & - & - & 1.67 \\
\hline
\end{tabular}

The relaxation time of birefringence depends on both the concentration and the electric field strength. The more concentrated, the longer was the relaxation time, whereas the relaxation time became noticeably shorter with increasing field strength. The latter effect is probably due to the strong interaction of oriented dipolar molecules. In this report, relaxation times obtained at the lowest field strength were adopted. In fact, they were measured at $E=27 \mathrm{esu} / \mathrm{cm}$.

\section{DISCUSSION}

As shown above, one could not observe any abrupt change in hydrodynamical, electrical and optical properties of PBLG in $m$-cresol solution with an increase of temperature. This strongly suggests that, in $m$-cresol, PBLG molecule retains its helical conformation up to $200^{\circ} \mathrm{C}$. At the same time, considerable decreases of the intrinsic viscosity, the average relaxation time, the dipole moment and the optical anisotropy factor suggest that, at high temperature, PBLG molecule becomes more flexible sustaining its helical conformation.

Tsvetkov, et al. ${ }^{16}$ have investigated the chain flexibility of PBLG in DMF, EDC and $m$-cresol, and have obtained a persistence length of $(1100 \pm$ 200) $\AA$ from sedimentation measurements, and $1200 \AA$ from flow birefringence measurements, respectively.

Wada and Kihara ${ }^{17}$ have calculated the persistence length of PBLG in $m$-cresol from dielectric dispersion measurements. Although they assumed that the stiffness of the helix is constant between 
25 and $90^{\circ} \mathrm{C}$, they estimated the persistence length of PBLG should be greater than $1500 \AA$ in $m$ cresol. Moha, et al., ${ }^{18}$ have investigated the light scattering and the relaxation time of birefringence of PBLG in DMF, EDC and $m$-cresol solution. They offered $900 \AA$ for the persistence length of PBLG on the assumption of $3_{10}$-helix.

From these, the authors have assumed that the persistence length of PBLG in $m$-cresol is $1200 \AA$ at $35^{\circ} \mathrm{C}$. Then one can use the equation of Kratky and Porod $^{19}$ for a wormlike chain

$$
\bar{\mu}^{2}=\frac{2 \mu_{0}^{2}\left(\mathrm{e}^{-x}-1+x\right)}{x^{2}}
$$

with

$$
x=\frac{L}{q}
$$

to estimate the persistence length $q$ at high temperatures from dipole moment data. In this equation, $\bar{\mu}^{2}$ is the mean square dipole moment of wormlike chain, $\mu_{0}$ is the dipole moment of perfectly extended chain. The contour length of PBLG is taken to be $1440 \AA$ from the molecular weight of the specimen and the $\alpha$-helix structure. Using the value of the dipole moment per unit length in DMF at $25^{\circ} \mathrm{C}$, one estimates the dipole moment of the perfectly extended chain to be $3.2 \times 10^{3} \mathrm{D}$. Results are summarized in the second column of Table III. Then, the rigidity $\varepsilon$ of the PBLG can be calculated by the use of a relation ${ }^{20}$

$$
\varepsilon=q k T
$$

Results are summarized in the fifth column of Table III.

The above results are based upon the assumption that the contribution of the side groups to the overall dipole moment does not change with the increase in temperature. Wada ${ }^{21}$ has confirmed that, at room temperature, side groups of PBLG are so oriented that their moments partially cancel the backbone dipole moment. Therefore, if one could estimate the change of the contribution of side chains to the overall dipole moment with temperature change, the persistence length of PBLG at high temperature would be estimated to be lower than this.

One can also estimate the persistence length from the relaxation time of birefringence. The rotatory diffusion constant $D$ of wormlike chain
Table III. Persistence length of PBLG in $m$ cresol from various methods

\begin{tabular}{rrrrr}
\hline \multirow{2}{*}{$\begin{array}{c}\text { Temp } \\
{ }^{\circ}\end{array}$} & \multicolumn{2}{c}{ Persistence length, $\AA$} & \\
\cline { 2 - 4 } & from $\mu$ & from $\bar{\tau}$ & from $[\eta]$ & \\
\hline 35 & 1200 & 700 & & \\
40 & & & 50,000 & \\
50 & 883 & 650 & & 3.18 \\
70 & & & 10,000 & \\
75 & 578 & 660 & & 2.15 \\
100 & 564 & 500 & 2,100 & 2.28 \\
125 & 407 & 400 & 1,080 & 1.78 \\
150 & 327 & 230 & 650 & 1.51 \\
170 & & 200 & & \\
175 & & & 400 & \\
200 & & & 270 & \\
\hline
\end{tabular}

is given by the equation ${ }^{22}$

$$
\begin{aligned}
D= & \frac{2 R T m^{2}}{M^{2} \eta_{0} q}\left[0.126\left(\frac{1}{q m}\right)^{1 / 2} M^{1 / 2}\right. \\
& \left.+\left(0.156 \ln \frac{2 q}{b}-0.387+0.16 \frac{b}{a}\right)\right]
\end{aligned}
$$

with

$$
a=\frac{\zeta}{3 \pi \eta_{0}}
$$

where $R$ is the gas constant, $M$ is the molecular weight, $m$ is the mass per unit length, $b$ is the spacing of the frictional elements, and $\zeta$ the frictional coefficient of the frictional element. Moha, et al. ${ }^{18}$ have shown plots of $M^{2} \eta_{0} D / k T$ against $M^{1 / 2}$ for several values of $q$, and one can use them to estimate the value of $q$ from the relaxation time. Results are summarized in Table III. They agree fairly well with those obtained from dipole moment data, in spite of the limited reliability of the data.

From the intrinsic viscosity, one can make another estimation of persistence length, by the use of Ullman's table. ${ }^{23}$ Results are also summarized in the fourth column of Table III. In this case, the persistence lengths of PBLG at low temperatures become too large, probably due to the inadequacy of the method in this case.

As shown in Table I, the optical anisotropy factor of PBLG in $m$-cresol also decreases with the increase of temperature. The optical anisotropy factor of a macromolecule in a medium is composed of the form birefringence and 
intrinsic birefringence. The former depends on the difference between the refractive indices of the medium and the solute, and on the shape of the solute molecule. The latter also depends on the shape of the solute molecule. On the assumption that the wormlike chain is bent in a plane with constant curvature as shown in Figure 4, and on the assumption of additivity of tensor components of optical polarizability, it can easily be shown (see Appendix) that the components of optical polarizability of bent chain become (provided that the chain lies in $X-Y$ plane)

$$
\begin{aligned}
& \gamma_{x x}=\frac{1}{2}\left(g_{x x}-g_{y y}\right) \frac{\sin \psi}{\psi}+\frac{1}{2}\left(g_{x x}+g_{y y}\right) \\
& \gamma_{y y}=\frac{1}{2}\left(g_{x x}-g_{y y}\right)\left(1-\frac{\sin \psi}{\psi}\right)+g_{y y} \\
& \gamma_{z z}=g_{z z}
\end{aligned}
$$

Where $g_{x x}$, etc., are the tensor components of the intrinsic optical polarizability of perfectly extended molecule, and $\psi$ is the central angle of the bent chain. Thus, if the orientation of the chain is restricted in a plane (in the $X-Y$ plane), the intrinsic optical anisotropy of a wormlike chain becomes simply

$$
\gamma_{x x}-\gamma_{y y}=\left(g_{x x}-g_{y y}\right) \frac{\sin \psi}{\psi}
$$

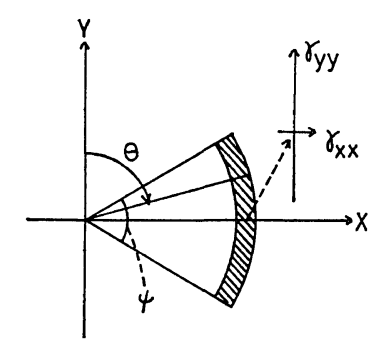

Figure 4. A bent rod in $X-Y$ plane. $Z$-axis is perpendicular to the plane of paper.

Thus the intrinsic optical anisotropy factor of a bent chain in one plane decreases in the same manner as the permanent dipole moment.

Theoretical treatment of the form birefringence is so complicated that, at present, quantitative discussion on the form birefringence of a bent rod is not possible. However, from the theory of Peterlin and Stuart, ${ }^{24}$ it is clear that the form birefringence of a wormlike particle would decrease with increase in flexibility.

On these bases, one can say that the decrease of optical anisotropy factor with the increase of temperature also supports the increase of the flexibility of PBLG helix at high temperature.

As to the reasons for the increasing flexibility of PBLG at high temperatures, one can mention three points:

1. At high temperature, thermal motion of side groups increases so that their stabilizing effect decreases.

2. The thermal motion of the main chain also increases at high temperature. This decreases the stiffness of the helix.

3. Thermal motion of solvent molecules also increases at high temperatures, so that they beat more vigorously the solute molecules, resulting in more pronounced bending of the helix.

\section{APPENDIX}

\section{Optical Anisotropy Factor of a Bent Rod}

As a first step of the calculation on the optical anisotropy factor of wormlike chain, the authors present here the calculation for a bent rod with constant curvature in a plane.

Let the rod lie in the $X-Y$ plane, the center of arc being on the origin of the coordinate system, as shown in Figure 4. Definitions of angle $\psi$ and $\theta$ are also given in Figure 4 .

Let $r$ be the radius of curvature, the contour length $L$ of rod is given by

$$
L=r \psi
$$

$\phi$ being expressed in radian.

If the straight rod is assumed to be axially symmetric, and the $X$-axis of cartesian coordinate system taken in the direction of the symmetric axis, then the components of optical polarizability tensor become

$$
\begin{aligned}
& g_{X X}=g_{/ \prime} \\
& g_{Y Y}=g_{Z Z}=g_{\perp} \\
& g_{X Y}=g_{Y X}=g_{X Z}=g_{Z X}=g_{Y Z}=g_{Z Y}=0
\end{aligned}
$$

where $g_{\| /}$and $g_{\perp}$ denote the optical polarizabilities of the straight rod parallel and perpendicular to the symmetry axis, respectively.

The line densities of polarizability are defined as follows: 


$$
\begin{aligned}
& \alpha_{\| l}=\frac{g_{\| \prime}}{L}=\frac{g_{\| \prime}}{r \psi} \\
& \alpha_{\perp}=\frac{g_{\perp}}{L}=\frac{g_{\perp}}{r \psi}
\end{aligned}
$$

Then, components of the optical polarizability density tensor at each part of rod are given by

$$
\begin{aligned}
& \alpha_{x x}=\left(\alpha_{/ /}-\alpha_{\perp}\right) \sin ^{2} \theta+\alpha_{\perp} \\
& \alpha_{y y}=\alpha_{/ /} \cos ^{2} \theta+\alpha_{\perp} \sin ^{2} \theta \\
& \alpha_{z z}=\alpha_{\perp} \\
& \alpha_{x y}=\alpha_{y x}=\left(\alpha_{/ /}-\alpha_{\perp}\right) \sin \theta \cos \theta \\
& \alpha_{x z}=\alpha_{z x}=\alpha_{y z}=\alpha_{z y}=0
\end{aligned}
$$

By the line integration along bent rod, one can obtain the optical anisotropy tensor of bent rod. In the coordinate system of this paper, one has

$$
\begin{aligned}
\gamma_{x x} & =\int_{-\frac{1}{2} \psi}^{\frac{1}{4} \psi} \alpha_{x x} r \mathrm{~d} \theta=\int_{-\frac{1}{2} \psi}^{\frac{1}{2} \psi}\left[\left(\alpha_{\|}-\alpha_{\perp}\right) \sin ^{2} \theta+\alpha_{\perp}\right] r \mathrm{~d} \theta \\
& =\frac{1}{2}\left(g_{\|}-g_{\perp}\right) \frac{\sin \psi}{\psi}+\frac{1}{2}\left(g_{\|}+g_{\perp}\right) \\
\gamma_{y y} & =\int_{-\frac{1}{2} \psi}^{\frac{1}{\psi} \psi} \alpha_{y y} r \mathrm{~d} \theta=\frac{1}{2}\left(g_{\|}-g_{\perp}\right)\left(1-\frac{\sin \psi}{\psi}\right)+g_{\perp} \\
\gamma_{z z} & =g_{\perp} \\
\gamma_{x y} & =\gamma_{y x}=\int_{-\frac{1}{2} \psi}^{\frac{1}{2} \psi} \alpha_{x y}=r \mathrm{~d} \theta=0
\end{aligned}
$$

Acknowledgments. The authors wish to express their hearty thanks to Professor K. Yoshioka for his suggestion, guidance, and kind support in elaboration of this paper.

\section{REFERENCES}

1. P. Doty and J. T. Yang, J. Amer. Chem. Soc., 78, 498 (1956).

2. Y. Hayashi, A. Teramoto, K. Kawahara, and H. Fujita, Biopolymers, 8, 403 (1969).
3. J. S. Franzen, J. B. Harry, and C. Bobik, Biopolymers, 5, 193 (1967).

4. H. Benoit, Ann. Phys., 6, 561 (1951).

5. C. T. O'Konski and B. H. Zimm, Science, 11, 113 (1950).

6. E. R. Blout and R. H. Karlson, J. Amer. Chem. Soc., 78, 941 (1956).

7. P. Doty, J. H. Bradbury, and A. M. Holtzer, ibid. 78, 947 (1956).

8. H. Watanabe, K. Yoshioka, and A. Wada, Biopolymers, 2, 91 (1964).

9. H. Watanabe, Nippon Kagaku Zasshi (J. Chem. Soc. Japan, Pure Chem. Sect.), 85, 403 (1964).

10. C. T. O'Konski, K. Yoshioka, and W. H. Orttung, J. Phys. Chem., 63, 1588 (1959).

11. K. Yoshioka and H. Watanabe, Nippon Kagaku Zasshi (J. Chem. Soc. Japan, Pure Chem. Sect.) 84, 626 (1963).

12. K. Yoshioka and H. Watanabe "Physical Principles and Techniques of Protein Chemistry, Part A," S. J. Leach, Ed., Academic Press, New York and London, 1969, pp 345-346.

13. S. Broersma, J. Chem. Phys., 32, 1626 (1960).

14. R. Simha, ibid. 13, 188 (1945).

15. V. Luzzati, M. Cesari, G. Spach, F. Masson, and J. M. Vincent, J. Mol. Biol., 3, 566 (1961).

16. V. N. Tsvetkov, I. N. Shtenirikova, E. I. Rymtsev, and V.S. Shazka, Vysokomol. Soedin., 7, 1098 (1965).

17. A. Wada and H. Kihara, Polymer J., 3, 482 (1972).

18. P. Moha, G. Weill, and H. Benoit, J. Chim. Phys., 61, 1240 (1564).

19. O. Kratky and G. Porod, Rec. Trav. Chem., 68, 1106 (1949).

20. N. Saito, K. Takahashi, and Y. Yunoki, $J$. Phys. Soc. Jap., 22, 219 (1967).

21. A. Wada, J. Mol. Biol., 3, 507 (1961).

22. J. E. Hearst, J. Chem. Phys., 38, 1062 (1963).

23. R. Ullman, ibid., 49, 5486 (1968).

24. A. Peterlin and H. A. Stuart, Z. Phys., 112, 129 (1939). 